\title{
Index Finger and Thumb Kinematics and Performance Measurements for Common Touchscreen Gestures
}

Deanna S. Asakawa ${ }^{a}$, Jack T. Dennerlein ${ }^{b}$ and Devin L. Jindrich ${ }^{a,}{ }^{\text {* }}$

${ }^{a}$ Department of Kinesiology, California State University San Marcos, 333. S. Twin Oaks Valley Rd., San Marcos, CA, USA

${ }^{\mathrm{b}}$ Department of Physical Therapy, Movement, and Rehabilitation Sciences, Bouvé College of Health Sciences, Northeastern University, 360 Huntington Avenue, Boston, USA

Keywords: Mobile technology, pinch, thumb

*Corresponding Author:

Devin L. Jindrich

Dept. of Kinesiology

California State University San Marcos

333 S. Twin Oaks Valley Rd.

San Marcos, CA 92096

$760750-7334$

djindrich@csusm.edu 


\section{Introduction}

Smart phones, mobile computing devices, and in-car navigation systems include touchscreens that require multi-touch finger taps, slides, and other gestures (Duggan and Rainie, 2012, Gold et al. , 2012, Kim and Song, 2014, Nacher et al. , 2015). Touchscreen technology could potentially contribute to repetitive strain injuries or other musculoskeletal disorders related to the overuse of the fingers and thumb (Berolo et al. , 2011, Gustafsson et al. , 2010, Jonsson et al. , 2011). For example, thumb joint osteoarthritis can occur from repetitive movement and result in pain and motion deficits (Fontana et al. , 2007, Gehrmann et al., 2010, Ming et al., 2006, Ugbolue et al. , 2005). Quantifying thumb and finger biomechanics during gestures can aid the design of touchscreen interfaces that are both easy to use and reduce strain on the musculoskeletal system (Trudeau et al. , 2013, Trudeau et al. , 2011, Young et al. , 2013).

Tapping on touchscreens or virtual keyboards may reduce exposure to forces and increase performance relative to conventional keyboards (Kim and Song, 2014, Kim et al. , 2014, Trudeau et al. , 2012). However, tapping may not be representative of the diverse gestures necessary for multitouch interaction. Multitouch gestures may involve greater joint excursions than tapping, potentially increasing the use of extended or flexed thumb and wrist joint postures associated with decreased performance (Trudeau, Young, 2012). Flicking and panning gestures used for in-vehicle navigation systems require more time than tapping, potentially increasing musculoskeletal exposure to loads (Kim and Song, 2014). However, zooming gestures that involve both the index finger and thumb require less time than tapping buttons when using a touchscreen map, suggesting that one- and two-fingered gestures may have important differences in some applications (Kim and Song, 2014). However, the performance and kinematics of most multitouch gestures have not been measured.

The aim of this study was to quantify performance and kinematics for common touchscreen gestures. Specifically, we measured task completion times and finger joint angles for seven different gestures that used either the index finger only, or both index finger and thumb. We hypothesized that index finger tapping would have the fastest task completion time of the gestures studied. We also hypothesized that two-finger gestures 
involving both the index finger and thumb would require longer task completion time and greater finger joint excursions than sliding gestures using only the index finger. Finally, we hypothesized that there would be differences among the four different directions of index finger sliding when comparing measured parameters.

\section{Methods}

We recruited eighteen unimpaired right-handed participants (9 female, 9 male; age 19-42 years) for the study. Subjects were recruited by word of mouth. No subject had a current upper limb injury or history of repetitive strain injury of the wrist, hand or fingers. All subjects had previously used touchscreen computing technology. The participants' average ( \pm SD) height was $1.67 \pm 0.08 \mathrm{~m}$ and average mass was $65.20 \pm 8.63 \mathrm{~kg}$. For all participants we measured hand length as the distance from the base of the palm to the tip of the third digit and hand width as the distance from the radial side of the second metacarpophalangeal joint to the ulnar side of the fifth metacarpophalangeal joint. Hand length and hand width were on average $18.0 \pm 1.1 \mathrm{~cm}$ and 8.6 $\pm 0.8 \mathrm{~cm}$ respectively. We obtained informed written consent from all participants using forms and protocols approved by California State University San Marcos Internal Review Board.

All gestures were completed on a Samsung Galaxy Tab 2 10.1-inch touchscreen tablet computer (Samsung Group, Seoul, South Korea). The touchscreen tablet measured 10.10 x 6.90 x 0.38 inches and weighed 1.28lb. A custom software application written for the Android platform displayed 6-cm square buttons for each gesture (Fig. 1). The 6-cm button size was chosen to represent a standard size slide or stretch commonly used for book reader or map touchscreen software applications. We used the same size button for all gestures to facilitate comparison among gestures. Participants were seated with the touchscreen mobile computing device placed in front of them in the landscape orientation flat on a table of height $0.91 \mathrm{~m}$. The long edge of the tablet computing device was placed parallel to the edge of the table at a distance of $0.1 \mathrm{~m}$. Participants were not allowed to adjust the position of the touchscreen tablet on the table. We also ensured that participants did not rest any part of their hand or wrist on the tablet computer or table. The participants 
3

4

completed sets of 11 gestures, presented in random order, for a total of 231 gestures. The gestures were: tapping with the index finger, sliding in four orthogonal directions on the touchscreen with the index finger (right, left, down, and up), pinch (pinching the index finger and thumb together as used for zoom out) and stretch (spreading the index finger and thumb apart as is used for zoom in). The 6-cm square buttons were centered in the middle of the touchscreen. Completion of a slide right gesture, for example, started on the left edge of the button, $3 \mathrm{~cm}$ left of the center of the screen, and ended on the right edge of the button which was $3 \mathrm{~cm}$ to the right of the center of the screen. The pinch and stretch gestures were completed with the index finger and thumb moving along a line that was at a $45^{\circ}$ angle from the long edge of the tablet computer. The participants completed all eleven repetitions of each gesture before moving on to the next gesture to minimize motion of the upper limb joints between repetitions. Participants were verbally instructed to complete each gesture as quickly as possible to make certain there were similar experimental conditions among subjects. No participant reported or was observed to have any fatigue after completing the study gestures. The custom Android software application recorded task completion time. Task completion times were measured bare-handed to ensure the experimental measurements were as close to real touchscreen use as possible.

Participants performed all gestures in two conditions: 1) bare-handed and 2) wearing a CyberGlove III 18-sensor system (CyberGlove Systems LLC, San Jose, CA). The CyberGlove III is reported by the manufacturer to have sensor resolution of $<1$ degree and sensor repeatability (average variability between wearings) of $3^{\circ}$. The CyberGlove recorded the joint angles of finger and thumb joints at a sampling rate of $30 \mathrm{~Hz}$ throughout all gestures. Specifically, we compared joint angle data for the index finger distal interphalangeal (DIP), proximal interphalangeal (PIP) and metacarpophalangeal (MCP) joints and the thumb interphalangeal (IP), metacarpophalangeal (MCP), and carpometacarpal (CMC) joints. The CyberGlove sensor system was calibrated for each subject by having them first lay their hand flat on the table surface with the fingers together and the thumb against the hand. This position established $0^{\circ}$ of flexion/extension of the finger and thumb joints. Additional calibration was then completed for the index finger and thumb joint angles as required by the 
CyberGlove software by having the subject touch the tips of the finger and thumb together (the OK sign). All

5 finger angles for the thumb and index finger were checked with a goniometer to ensure the joint angles were

7 accurate and that the thumb and index finger tips were touching. We then had all subjects perform $90^{\circ}$ of index

finger MCP joint flexion as measured with a goniometer and compared the value to the CyberGlove joint angle

reading to determine accuracy. Calibration was repeated if necessary to ensure accurate joint angle

measurements. The set of all gestures was completed twice, once bare-handed and once with the subject

wearing the CyberGlove. A counter-balance design was used such that some participants started with the barehanded condition and others started with wearing the CyberGlove. Subjects with hand length less than $17.0 \mathrm{~cm}$

were not able to actuate the gesture buttons while wearing the CyberGlove joint angle sensors. Therefore, we successfully measured finger joint angles for 14 of the 18 participants.

Statistical analysis of data including analysis of variance (ANOVA) and post-hoc tests were completed using SPSS Statistics software (IBM Inc., New York, NY). We performed mixed-effects ANOVA, with participant as the between-subject factor and gesture as the within-subject factor. To determine significant differences, we used Bonferroni and Tukey's post hoc tests for equal variances, and Games-Howell post hoc test for measurements that demonstrated unequal variances or unequal number of successfully completed gestures.

\section{Results}

\subsection{Index finger tapping demonstrated faster task completion times than other gestures studied}

Mean ( \pm standard deviation) task completion time for index finger tapping was $567 \pm 190 \mathrm{~ms}$ (Fig. 2).

Tapping with the index finger had a significantly faster task completion time than all other gestures $(\mathrm{p}<0.001)$.

Therefore, the data support our performance hypothesis as tapping was the quickest gesture to complete.

\subsection{Two finger gestures had faster task completion times than single-finger sliding gestures}


The two-finger pinch gesture, with completion time of $765 \pm 277 \mathrm{~ms}$, had significantly faster completion times than all four directions of single-finger sliding gestures ( $<<0.001$; Fig. 2). Moreover, even though the twofinger stretch gesture was significantly slower at $843 \pm 317 \mathrm{~ms}$ than two-finger pinch ( $<<0.001)$, two-finger stretch was significantly faster than slide left $(945 \pm 276 \mathrm{~ms}$, $\mathrm{p}<0.01)$, slide down, $948 \pm 257 \mathrm{~ms}$, and slide up (998 $\pm 341 \mathrm{~ms}, \mathrm{p}<0.001)$. We did not observe significant differences between two-finger stretch and one-finger slide right. Based on the majority of comparisons, we reject the hypothesis that two-finger gestures take longer to complete than single-finger sliding gestures.

\subsection{Gestures using two fingers involved greater finger joint excursions than single-finger gestures}

Joint excursions measured for the index finger and thumb support the hypothesis that gestures using two fingers, such as pinch and stretch, involve greater joint excursions than single finger gestures (Table 1). However, comparisons within gestures showed differences in excursions among the three finger joints during gestures. For example, index finger MCP excursions were $44 \%$ to $148 \%$ percent more than PIP excursions during single-finger gestures (Table 1). Similarly, index finger MCP excursions were $85 \%$ to $234 \%$ percent more than PIP excursions during two-finger gestures. Across all gestures, the DIP joint moved a maximum of 3 $\pm 5^{\circ}$, and DIP joint movement was not significantly different among gestures. Because the index finger MCP joint exhibited larger excursions than the PIP joints, we focus our comparison of gestures to MCP joint movement.

MCP joint excursions were different among gestures. The maximum index finger MCP joint flexion, $66.3 \pm 8.6^{\circ}$, and greatest excursion of the index finger MCP joint, $63.2 \pm 16.0^{\circ}$, were recorded for the two-finger stretch gesture (Table 1). Index finger MCP joint excursion during stretch was $86 \%$ to $177 \%$ greater than MCP joint excursion during single-finger gestures. Stretch had significantly greater MCP joint excursion when compared to all single-finger gestures $(\mathrm{p}<0.01$; Table 1$)$. For pinch, excursion of the MCP joint was significantly greater than tap, slide right and slide up ( $\mathrm{p}<0.01$; Table 1). However, stretch joint excursion was 
5

8

Sliding to the right with a task completion time of $880 \pm 199 \mathrm{~ms}$, was significantly faster than slide left, down, and slide up ( $\mathrm{p}<0.01 ;$ Fig. 2). Neither MCP nor PIP joint excursions were significantly different among the four directions of sliding (Table 1). Overall, our data support the hypothesis that single-finger sliding gestures in different directions differ in completion time, but not in overall joint excursions.

\section{Discussion}

The purpose of this study was to test the hypothesis that one- and two-finger touchscreen gestures differ in joint movement extent and completion time on the tablet surface. Specifically, we compared two-finger gestures, pinch and stretch, to one-finger tapping and sliding in four directions. This study contributes to the understanding of gestural touchscreen interactions by providing quantitative comparisons of seven popular gestures. In all, we found significant differences among the gestures. Contrary to our hypothesis, gestures requiring use of both the index finger and thumb did not take longer to complete compared to gestures using the index finger alone. However, the stretch gesture (zoom in) did show the greatest joint excursions. No single gesture studied had the smallest value for both task completion time and finger joint excursion.

Several limitations of our study should be taken into account when interpreting our results. First, we measured task completion time as the time from when subjects hit a prompt button until they had completed the gesture. Consequently, the task completion times reported here are potentially longer than if measured from the start of the gesture measured using a different metric. However, identifying the beginning of a gesture can be challenging, as arm, hand, and finger movements may be continuous and not discrete. Using the prompt provided an unambiguous measure of starting time. Moreover, we do not expect that the time to press the 
prompt button is different for different gestures. Therefore, we expect the prompt to represent a constant bias and not affect the ordering of completion times among gestures.

Second, we did not constrain the distance the participants moved their fingers to complete the sliding gestures. Specifically, the participants could perform a larger gesture than what was necessary to simply complete the slide gesture, potentially leading to longer task completion times. The tap and pinch gestures were 14

Third, we measured only the finger and thumb joint angles, and no other upper limb angles. Movement at other joints, including the wrist, the elbow or the shoulder, could contribute to completing these gestures. Measurement of arm and body motion was beyond the scope of this study, but future studies will evaluate the contributions of arm and body movement to multitouch gestures.

Fourth, we studied movements on a relatively large touchscreen and not on the smaller form factor typical of smart phones. The 6-cm square button is a size that could be representative of some gestures on smaller hand-held mobile computing devices as well. Also, due to the size of the device, we placed the touchscreen tablet flat on a table to control variability and eliminate the effects of variable holding postures on performance and kinematics. Holding a device with one hand while interacting with a touchscreen may be more difficult and could decrease performance(Trudeau et al. , 2016). Additional studies will be necessary to investigate the effects of button size, touchscreen size, and device position on gesture performance. 
Finally, the CyberGlove III sensor could not be used by participants with hand length less than $17.0 \mathrm{~cm}$.

Subjects with small hands were not able to complete the gesture buttons as the glove fingertip interfered with the ability to effectively actuate the touchscreen buttons. A hand length of $17.0 \mathrm{~cm}$ is below the $50^{\text {th }}$ percentile for hand size (Pereira et al. , 2013). The hand size limitation is unlikely to affect the conclusions of our study because there was no correlation between hand size and joint excursion measurements for any of the seven gestures. Moreover, accuracy of a CyberGlove sensor can be affected by hand size due to the resulting placement of the sensors relative to each joint (Buffi et al. , 2014). Therefore, excluding measurements from participants with small hands was necessary to ensure valid joint angle measurements.

Our findings contribute to a more general understanding of touchscreen gestures. For example, index finger sliding gestures had longer task completion times than index finger tapping, consistent with previous studies that showed flicking had a longer task completion time than tapping (Kim and Song, 2014). However, we found index finger tapping to have significantly faster task completion time than all other gestures studied. Our results conflict with data reporting that less time was required to zoom a map using stretch and pinch gestures compared to tapping touch buttons for in-vehicle navigation systems (Kim and Song, 2014). The conflict between our findings and those of Kim and Song (2014) could in part be due to differences in gesture tasks, visual tasks, and the experimental set up between studies. For the in-vehicle touchscreen tasks used by Kim and Song (2014), the touchscreen was angled at $75^{\circ}$ and was placed to the right of the subject at a distance of $700 \mathrm{~mm}$. In our study, the touchscreen was placed directly in front of the participant and the only visual and gesture task was gesture completion. Moreover, all gestures were completed with same size gesture buttons so that we could control the gesture size and compare among like-size gestures. The set up and task differed from the Kim and Song (2014) study in which participants had to look to the right, reach, and complete a series of gestures for which an average unit operation time per gesture was calculated. Therefore, the specific tablet computing device placement, gesture task, and different calculation of gesture completion time may influence two-fingered gesture completion time results. 
Task completion times may be dependent on the distance traveled by the fingertip when evaluating gestures for use in touchscreen software applications (Kim and Song, 2014). For example, in this study tap was the fastest gesture to complete, as expected. The tap gesture does not require the finger to travel a distance over the touchscreen as do sliding, pinch, and stretch gestures. Two-finger pinch gestures may have demonstrated shorter task completion times compared to other gestures because of a shorter distance traveled to complete a pinch or stretch compared to sliding gestures. The two-finger gestures were completed on the diagonal of the 6$\mathrm{cm}$ square button; each finger traversed $4.2 \mathrm{~cm}$ to successfully complete the gesture task. In the sliding gestures, the entire 6-cm length of the button was traversed in each gesture. Hence, the distance the index finger and thumb traveled for sliding was 30\% greater than the distance traveled by each of the fingers for pinch and stretch. We chose the 6-cm button size to represent a typical slide to turn a page on a book reader touchscreen software application. However, completion times for a 6-cm square button may not represent real usage conditions in which distance traveled, task completion times for gestures and factors such as posture are more variable (Dennerlein, 2015, Gold, Driban, 2012). The most proximal joints of the thumb and index finger experienced the greatest joint angle excursion during gesturing. Pinch and stretch gestures involved the most motion of the thumb CMC and index finger MCP joint. Chronic wear of the thumb CMC joint has been linked to incidence of osteoarthritis (Jonsson, Johnson, 2011, Ming, Pietikainen, 2006, Ugbolue, Hsu, 2005). Based simply on joint excursion measures, the pinch and stretch gestures could potentially increase exposure to motion and consequently increase wear on proximal joints of the index finger and thumb compared to the single-finger gestures studied. Furthermore, the finding that the index finger DIP joint flexes a maximum of $3^{\circ}$ during the gestures studied along with the joint excursion comparisons presented could help with hand surgery planning, such as determining joint angle for thumb joint arthrodesis, for those who use smartphones and touchscreens regularly (Yao et al. , 2012).

This study contributes several findings with possible practical importance to touchscreen interface design. There is great variability of postures when using touchscreen mobile computing devices (Ciccarelli et al. 
2015, Gold, Driban, 2012). Moreover, there is currently limited knowledge of important factors such as muscle activity for even the most common postures (Kietrys et al. , 2015). Therefore, developing best practices for mobile computing technology is in its infancy (Dennerlein, 2015). Quantitative measures of touchscreen performance and kinematics will contribute to the development of recommendations for best practices.

Quantitative joint angle data may guide touchscreen software designers to select gestures that reduce joint excursions. Also, task completion times could be used in design to aid selection of gestures that minimize muscle activity and exposure to force. Quantitative measures can thereby possibly be used to reduce the amount of strain on the musculoskeletal system.

Posture and muscle activity are important for predicting musculoskeletal complaints and disorders (Dennerlein, 2015). Quantitative measures of joint angles during touchscreen gestures allows us to evaluate gestures such as stretch that have greater motion of the MCP joint and could possibly contribute to greater wear on the joint. Software interfaces that require fewer pinch and stretch gestures or smaller pinch and stretch gestures may potentially reduce strain on the index finger and thumb joints. Gestures such taps and slides may reduce musculoskeletal strain as the joint postures of the fingers are more neutral, avoiding excessive flexion or extension of the finger joints.

Gestures that can be completed more quickly may increase ease of use. For example, using taps could improve interface performance relative to using sliding gestures, pinch or stretch. Based on our results, we hypothesize that tapping could reduce joint excursion at the index finger MCP joint relative to pinch or stretch. Tapping a single button could be used to zoom in or zoom out (Kim and Song, 2014). Other tasks such as page turning on a touchscreen book reader can also be accomplished with taps rather slides, and could potentially reduce strain on the musculoskeletal system and provide greater ease of use.

In conclusion, we found that one- and two-fingered gestures differed in several respects. Tapping with the index finger had significantly faster task completion time than all other gestures. However, participants 
could complete the two-fingered pinch gesture more quickly than index finger sliding, despite the greater joint excursion needed for two-fingered gestures. The two-finger stretch gesture demonstrated greater joint excursion at the MCP joint than all other gestures studied. In addition, comparison among the four directions of sliding gestures showed differences in task completion time. This study provides a first step to comparing index finger tapping, sliding and two-finger gestures used to zoom an image in or out. Quantitative information about touchscreen gestures could be useful for developing further understanding of risk factors for repetitive touchscreen use and potential strategies for reducing musculoskeletal injury. Also, the quantitative comparisons of finger joint kinematics, and completion times for gestures could help aid in the creation of guidelines for best touchscreen use practices and design of touchscreen software interfaces that incorporate performance and joint kinematic considerations to benefit users. 


\section{References}

Berolo S, Wells RP, Amick BC, 3rd. Musculoskeletal symptoms among mobile hand-held device users and their relationship to device use: A preliminary study in a Canadian university population. Appl Ergon. 2011;42:371-8.

Buffi JH, Sancho Bru JL, Crisco JJ, Murray WM. Evaluation of hand motion capture protocol using static computed tomography images: application to an instrumented glove. J Biomech Eng. 2014;136:124501.

Ciccarelli M, Chen JD, Vaz S, Cordier R, Falkmer T. Managing children's postural risk when using mobile technology at home: Challenges and strategies. Applied Ergonomics. 2015;51:189-98.

Dennerlein JT. The state of ergonomics for mobile computing technology. Work. 2015;52:269-77.

Duggan M, Rainie L. Cell Phone Activities Pew Research Center's Internet \& American Life Project2012. p. 20.

Fontana L, Neel S, Claise J-M, Ughetto S, Catilina P. Osteoarthritis of the Thumb Carpometacarpal Joint in Women and Occupational Risk Factors: A Case-Control Study. The Journal of Hand Surgery. 2007;32:459-65.

Gehrmann SV, Tang J, Li ZM, Goitz RJ, Windolf J, Kaufmann RA. Motion deficit of the thumb in CMC joint arthritis. J Hand Surg Am. 2010;35:1449-53.

Gold JE, Driban JB, Thomas N, Chakravarty T, Channell V, Komaroff E. Postures, typing strategies, and gender differences in mobile device usage: An observational study. Applied Ergonomics. 2012;43:408-12.

Gustafsson E, Johnson PW, Hagberg M. Thumb postures and physical loads during mobile phone use - A comparison of young adults with and without musculoskeletal symptoms. Journal of Electromyography and Kinesiology. 2010;20:127-35.

Jonsson P, Johnson PW, Hagberg M, Forsman M. Thumb joint movement and muscular activity during mobile phone texting - A methodological study. Journal of Electromyography and Kinesiology. 2011;21:363-70.

Kietrys DM, Gerg MJ, Dropkin J, Gold JE. Mobile input device type, texting style and screen size influence upper extremity and trapezius muscle activity, and cervical posture while texting. Applied Ergonomics. 2015;50:98-104.

Kim H, Song H. Evaluation of the safety and usability of touch gestures in operating invehicle information systems with visual occlusion. Appl Ergon. 2014;45:789-98.

Kim JH, Aulck L, Bartha MC, Harper CA, Johnson PW. Differences in typing forces, muscle activity, comfort, and typing performance among virtual, notebook, and desktop keyboards. Appl Ergon. 2014;45:1406-13.

Ming Z, Pietikainen S, Hänninen O. Excessive texting in pathophysiology of first carpometacarpal joint arthritis. Pathophysiology. 2006;13:269-70.

Nacher V, Jaen J, Navarro E, Catala A, González P. Multi-touch gestures for pre-kindergarten children. International Journal of Human-Computer Studies. 2015;73:37-51.

Pereira A, Miller T, Huang Y, Odell D, Rempel D. Holding a tablet computer with one hand: effect of tablet design features on biomechanics and subjective usability among users with small hands. Ergomonics. 2013. 
Trudeau MB, Asakawa DS, Jindrich DL, Dennerlein JT. Two-handed grip on a mobile phone affords greater thumb motor performance, decreased variability, and a more extended thumb posture than a one-handed grip. Applied Ergonomics. 2016;52:24-8.

Trudeau MB, Catalano PJ, Jindrich DL, Dennerlein JT. Tablet Keyboard Configuration Affects Performance, Discomfort and Task Difficulty for Thumb Typing in a Two-Handed Grip. PLoS One. 2013;8:e67525.

Trudeau MB, Udtamadilok T, Karlson AK, Dennerlein JT. Thumb Motor Performance Varies by Movement Orientation, Direction, and Device Size During Single-Handed Mobile Phone Use. Human Factors: The Journal of the Human Factors and Ergonomics Society. 2011;54:52-9.

Trudeau MB, Young JG, Jindrich DL, Dennerlein JT. Thumb motor performance varies with thumb and wrist posture during single-handed mobile phone use. J Biomech. 2012;45:2349-54.

Ugbolue UC, Hsu W-H, Goitz RJ, Li Z-M. Tendon and nerve displacement at the wrist during finger movements. Clinical Biomechanics. 2005;20:50-6.

Yao J, Park MJ, Davis D, Chang J. Ideal position for thumb interphanlangeal arthodesis in the era of smartphones and text communication. Orthopedics. 2012;35:3.

Young JG, Trudeau MB, Odell D, Marinelli K, Dennerlein JT. Wrist and shoulder posture and muscle activity during touch-screen tablet use: effects of usage configuration, tablet type, and interacting hand. Work. 2013;45:59-71. 


\section{Figure Captions}

Figure 1. Experimental apparatus. Participants were seated with the tablet computing device placed on the table in front of them. Each participant actuated 6-cm square buttons displayed with custom software for each gesture type while wearing the CyberGlove sensor to measure finger joint angles (task completion time and fingertip pressure were measured during barehanded gesture completion). Arrows on the buttons illustrated the direction of finger movement for each gesture.

Figure 2. Average task completion time (ms) for barehanded gestures. Errors bars represent one standard deviation. Single-finger gestures were completed with the index finger. Two-finger gestures were completed with the index finger and thumb. Post hoc testing results are denoted with letters for significant difference: $t$ for tap $(\mathrm{p}<0.001), \mathrm{r}$ for slide right $(\mathrm{p}<0.01), 1$ for slide left $(\mathrm{p}<0.01), \mathrm{d}$ for slide down $(\mathrm{p}<0.05), \mathrm{u}$ for slide up $(\mathrm{p}<0.05)$, and $\mathrm{p}$ for pinch $(\mathrm{p}<0.001)$. 
Figure 1.

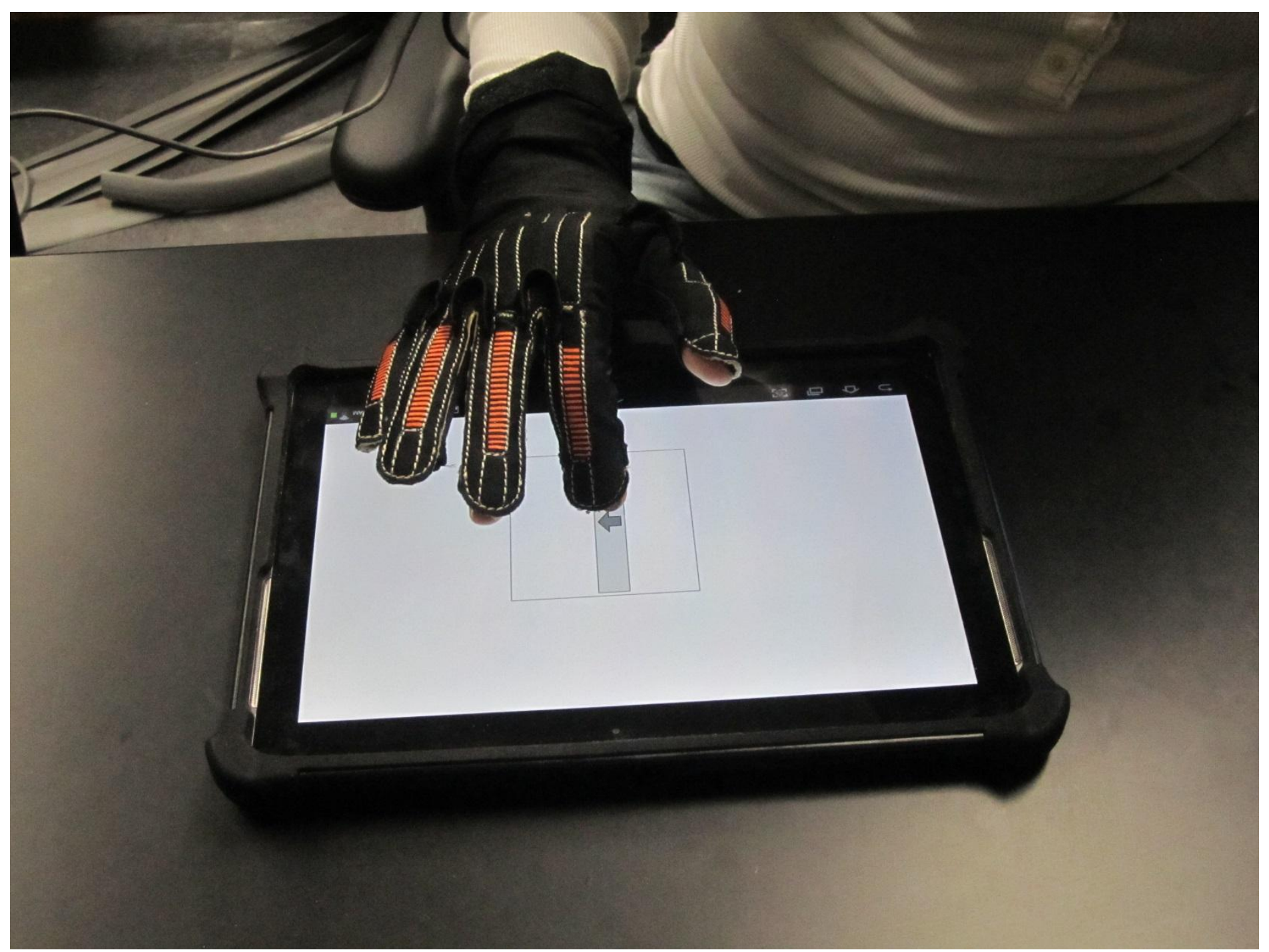


Figure 2.

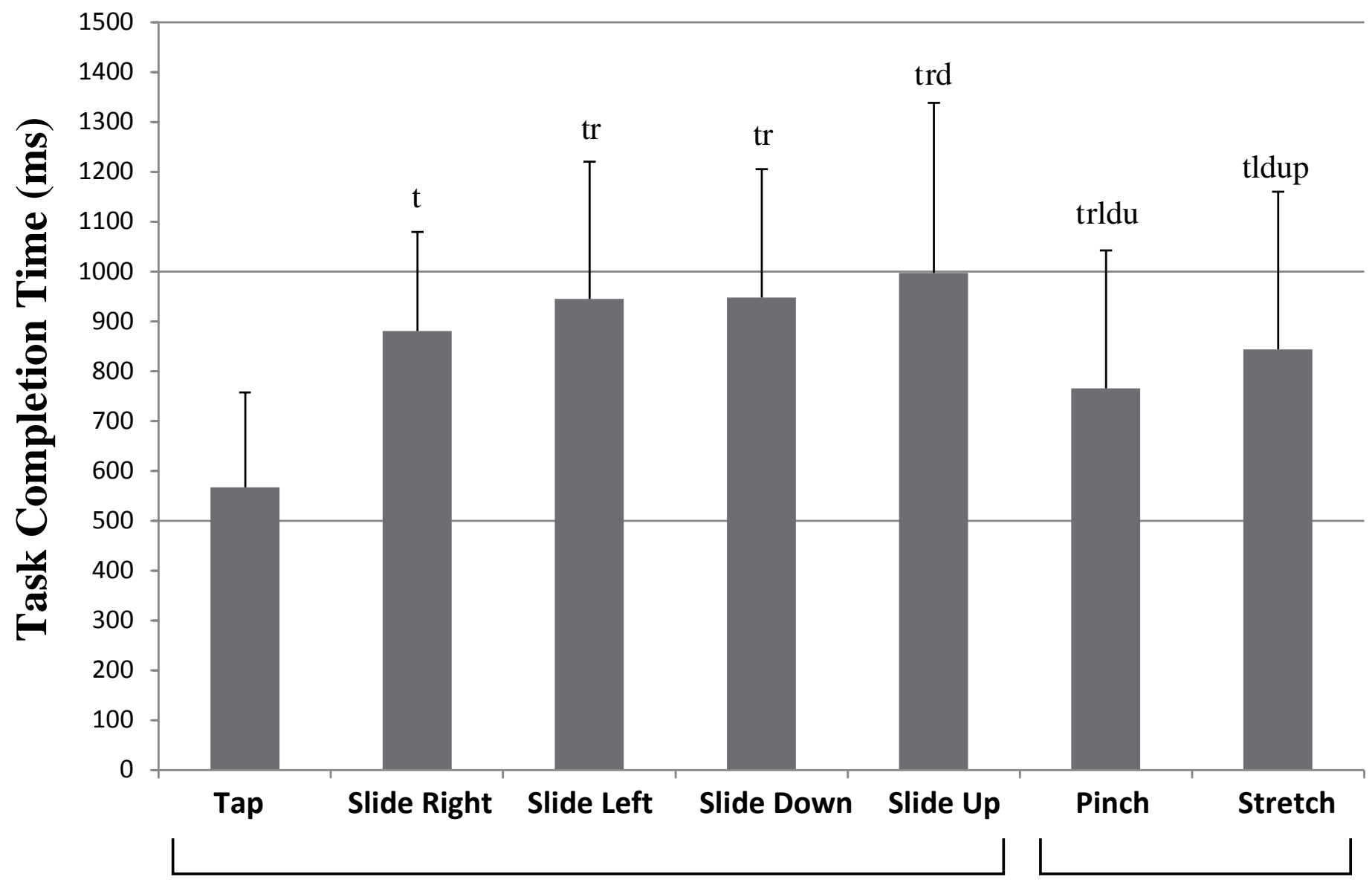

Single-finger

Two-finger 


\section{Tables:}

Table 1. Finger joint angles in degrees for each gesture averaged for 14 participants. Joint angle minimum (min) and maximum $(\max )$ flexion/extension $(\mathrm{F} / \mathrm{E})$ value (and standard deviation) and joint excursion $(\mathrm{EXC})$ are reported for the index finger PIP, and MCP joints, and the thumb IP, MCP, and CMC joints.

Abduction/adduction ( $\mathrm{AB} / \mathrm{AD}$ ) of the thumb $\mathrm{CMC}$ is also reported. Negative (positive) values indicate joint flexion (extension). All joint angles are relative to the hand flat position used for calibration. Significant differences for post hoc comparisons in index finger MCP joint excursions are indicated by superscripts.

\begin{tabular}{|c|c|c|c|c|c|c|c|}
\hline \multirow[t]{2}{*}{ Gesture } & & \multirow{2}{*}{$\begin{array}{l}\text { Index } \\
\operatorname{PIP}\left({ }^{\circ}\right) \\
\end{array}$} & \multirow{2}{*}{$\begin{array}{l}\text { Finger } \\
M C P\left({ }^{\circ}\right) \\
\end{array}$} & \multicolumn{4}{|l|}{ Thumb } \\
\hline & & & & $\mathrm{IP}\left({ }^{\circ}\right)$ & $\mathrm{MCP}\left({ }^{\circ}\right)$ & CMC F/E ( $\left.{ }^{\circ}\right)$ & $C M C A B / A D\left({ }^{\circ}\right)$ \\
\hline \multirow[t]{3}{*}{ Tap } & $\min$ & $-12(15)$ & $-45(10)$ & & & & \\
\hline & $\max$ & $1(2)$ & $-19(13)$ & & & & \\
\hline & $\mathrm{EXC}$ & $13(15)$ & $27(13)^{\mathbf{s}, \mathbf{p}}$ & & & & \\
\hline \multirow[t]{3}{*}{ Slide Right } & $\min$ & $-15(12)$ & $-48(17)$ & & & & \\
\hline & $\max$ & $1(2)$ & $-26(10)$ & & & & \\
\hline & $\mathrm{EXC}$ & $16(12)$ & $22(15)^{\mathbf{s , p}}$ & & & & \\
\hline \multirow[t]{3}{*}{ Slide Left } & $\min$ & $-10(9)$ & $-50(19)$ & & & & \\
\hline & $\max$ & $1(1)$ & $-21(12)$ & & & & \\
\hline & $\mathrm{EXC}$ & $10(9)$ & $29(22)^{\mathrm{s}}$ & & & & \\
\hline \multirow[t]{3}{*}{ Slide Up } & $\min$ & $-15(17)$ & $-48(11)$ & & & & \\
\hline & $\max$ & $1(2)$ & $-18(11)$ & & & & \\
\hline & $\mathrm{EXC}$ & $16(17)$ & $30(10)^{\mathbf{s , p}}$ & & & & \\
\hline \multirow[t]{3}{*}{ Slide Down } & Min & $-13(14)$ & $-55(10)$ & & & & \\
\hline & Max & $1(1)$ & $-21(12)$ & & & & \\
\hline & EXC & $14(14)$ & $34(10)^{\mathrm{s}}$ & & & & \\
\hline \multirow[t]{3}{*}{ Pinch } & $\min$ & $-23(19)$ & $-66(11)$ & $-22(11)$ & $-18(31)$ & $-80(34)$ & $-21(7)$ \\
\hline & $\max$ & $1(1)$ & $-21(14)$ & $13(21)$ & $14(20)$ & $-30(27)$ & $-10(6)$ \\
\hline & $\mathrm{EXC}$ & $25(19)$ & $46(11)^{s}$ & 35 (18) & $31(18)$ & $51(23)$ & $11(6)$ \\
\hline \multirow{3}{*}{ Stretch } & $\min$ & $-18(14)$ & $-66(9)$ & $-17(10)$ & $-15(30)$ & $-79(30)$ & $-21(10)$ \\
\hline & $\max$ & $1(1)$ & $-3(18)$ & $18(16)$ & $24(23)$ & $-21(22)$ & $-10(6)$ \\
\hline & $\mathrm{EXC}$ & $19(14)$ & $63(16)^{p}$ & $36(19)$ & $39(17)$ & $56(18)$ & $11(7)$ \\
\hline
\end{tabular}

$\mathrm{p}=$ significantly different from pinch $(\mathrm{p}<0.01)$

$\mathrm{s}=$ significantly different from stretch $(\mathrm{p}<0.01)$ 